\title{
Star formation laws in extreme starbursts
}

\author{
S. García-Burillo ${ }^{1}$, A. Usero ${ }^{1}$ and A. Alonso-Herrero ${ }^{2}$ \\ ${ }^{1}$ Observatorio de Madrid (OAN-IGN) \\ Alfonso XII, 3, 28014-Madrid, Spain \\ email: s.gburillo@oan.es, a.usero@oan.es \\ ${ }^{2}$ Instituto de Física de Cantabria (IFCA-UC) \\ 39005-Santander, Spain \\ email: aalonso@ifca.unican.es
}

\begin{abstract}
The observational study of star formation laws is paramount to disentangling the physical processes at work on local and global scales in galaxies. To this aim we have expanded the sample of extreme starbursts, represented by local LIRGs and ULIRGs, with high-quality data obtained in the 1-0 line of HCN. The analysis of the new data shows that the star formation efficiency of the dense molecular gas, derived from the FIR/HCN luminosity ratio, is a factor 3-4 higher in extreme starbursts compared to normal galaxies. We find a duality in the KennicuttSchmidt laws that is enhanced if we account for the different conversion factor for $\operatorname{HCN}\left(\alpha_{\mathrm{HCN}}\right)$ in extreme starbursts and correct for the unobscured star formation rate in normal galaxies. We find that it is possible to fit the observed differences in the FIR/HCN ratios between normal galaxies and LIRGs/ULIRGs with a common constant star formation rate per free-fall time $\left(\mathrm{SFR}_{\mathrm{ff}}\right)$ if we assume that HCN densities are $\sim 1-2$ orders of magnitude higher in LIRGs/ULIRGs, and provided that $\mathrm{SFR}_{\mathrm{ff}} \sim 0.005-0.01$ and/or if $\alpha_{\mathrm{HCN}}$ is a factor of a few lower than our favored values.
\end{abstract}

Keywords. ISM: molecules — galaxies: starburst — galaxies: ISM — radio lines: galaxies

\section{Star formation laws in galaxies: an observational perspective}

Based on simple theoretical arguments, Schmidt (1959) proposed that the star formation rate volume density $\left(\rho_{\mathrm{SFR}}\right)$ in galaxies should scale as a power law of the corresponding gas volume density $\left(\rho_{\text {gas }}\right): \rho_{\mathrm{SFR}} \propto \rho_{\text {gas }}^{n}$. If the relevant time scale for star formation is the local free-fall time $\left(\mathrm{t}_{\mathrm{ff}}\right)$, then $\rho_{\mathrm{SFR}} \propto \rho_{\text {gas }} / t_{\mathrm{ff}} \propto \rho_{\text {gas }}^{1.5}$. A similar relation between the surface densities $\left(\Sigma_{\mathrm{SFR}}, \Sigma_{\text {gas }}\right)$ follows provided that the gas scale-height remains constant in different galaxy populations, a widely adopted yet far from trivial assumption.

Observers have used different proxies for $\Sigma_{\mathrm{SFR}}$ and $\Sigma_{\text {gas }}$ to derive star formation relations in normal galaxies and extreme starbursts. Kennicutt et al. (1998) found a power law index $n=1.40 \pm 0.15$ using a compilation of galaxy-averaged $\mathrm{CO}+\mathrm{HI}$ data obtained in a sample of $\sim 100$ galaxies. More recently the CO surveys of Daddi et al. (2010) and Genzel et al. (2010) presented evidence of bimodality in galaxy-averaged star formation laws: normal galaxies show 4-10 times longer depletion time-scales compared to mergers. Observations of HCN lines have also been used to study the star formation laws for the dense molecular gas. The first studies by Gao \& Solomon (2004) found that the star formation efficiency measured with respect to the dense molecular gas content $\left(\mathrm{SFE}_{\text {dense }} \propto L_{\mathrm{IR}} / L_{\mathrm{HCN}(1-0)}^{\prime}\right)$ was about constant in galaxies. This scenario was later questioned by Graciá-Carpio et al. (2008), who observed a sample of LIRGs/ULIRGs and found that $\mathrm{SFE}_{\text {dense }}$ is a factor $\sim 2-3$ higher in IR luminous targets compared to normal galaxies. These observations suggested that galaxies classified as LIRGs may represent the transition point in the star formation laws. To improve the statisitics in 
this luminosity range, we have recently expanded up to 28 the number of LIRGs with high-quality HCN data obtained with the IRAM 30m telescope. Out of these, fourteen were extracted from the sample of nearby LIRGs studied by Alonso-Herrero et al. (2006). The results of this work, published by García-Burillo et al. (2012), have been used to study if the observed bimodality of star formation laws in galaxies derived from $\mathrm{CO}$ can be extended to the higher density regime probed by HCN. We have also compared our observations with the predictions of theoretical models in which the efficiency of star formation is determined by the ratio of a constant star formation rate per free-fall time $\left(\mathrm{SFR}_{\mathrm{ff}}\right)$ to the local free-fall time $\mathrm{t}_{\mathrm{ff}}$ (Krumholz \& McKee 2005).
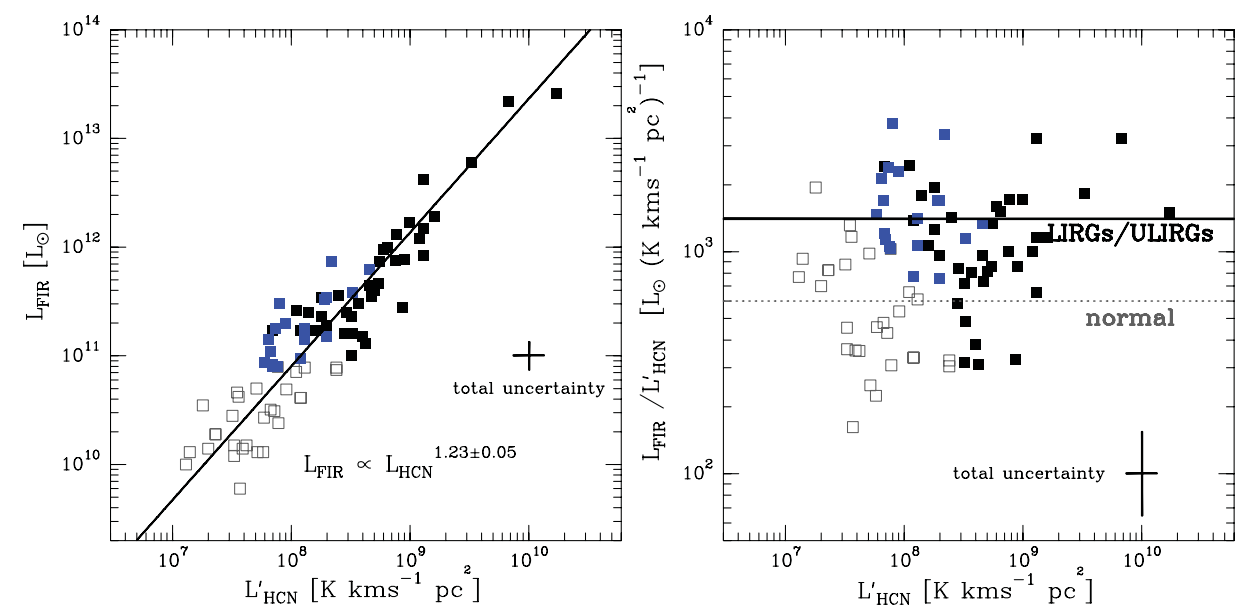

Figure 1. a) (left panel) $L_{\mathrm{FIR}}-L_{\mathrm{HCN}(1-0)}^{\prime}$ correlation plot derived for different galaxy samples. The solid line represents the orthogonal regression fit to the full sample. b) (right panel) The $L_{\mathrm{FIR}} / L_{\mathrm{HCN}(1-0)}^{\prime}$ luminosity ratio as a function of $L_{\mathrm{HCN}}^{\prime}$. We display with different symbols normal galaxies $\left(L_{\mathrm{IR}}<10^{11} L_{\odot}\right.$, open squares) and luminous infrared galaxies $\left(L_{\mathrm{IR}}>10^{11} L_{\odot}\right.$, filled squares). The data from the new sample of LIRGs is identified by blue color markers. The horizontal lines indicate the average value of $L_{\mathrm{FIR}} / L_{\mathrm{HCN}(1-0)}^{\prime}$ in normal galaxies $\left(\sim 600 \pm 70 L_{\odot} /\left(\mathrm{K} \mathrm{km} \mathrm{s}^{-1} \mathrm{pc}^{2}\right)\right)$ and LIRGs/ULIRGs $\left(\sim 1400 \pm 100 L_{\odot} /\left(\mathrm{K} \mathrm{km} \mathrm{s}^{-1} \mathrm{pc}^{2}\right)\right)$.

\section{Star formation laws of the dense gas: a new sample of LIRGs}

As illustrated in Fig. 1a, with the addition of the new LIRG sample we confirm that the $L_{\mathrm{FIR}}-L_{\mathrm{HCN}(1-0)}^{\prime}$ correlation is superlinear (power law index $\sim 1.23 \pm 0.05$ ). Furthermore we corroborate on a more solid statistical basis, compared to the work of Graciá-Carpio et al. (2008), that the average star formation efficiency of the dense gas is a factor $\sim 2-3$ higher in LIRGs/ULIRGs $\left(\sim 1400 \pm 100 L_{\odot} /\left(\mathrm{Kkms}^{-1} \mathrm{pc}^{2}\right)\right)$ compared to normal galaxies $\left(\sim 600 \pm 70 L_{\odot} /\left(\mathrm{Kkms}^{-1} \mathrm{pc}^{2}\right)\right)$ (See Fig. 1b). If we use universal conversion factors for $\mathrm{HCN}$ and FIR luminosities, a fit to the full sample yields a power index $n=1.12 \pm 0.04$ for the implied Kennicutt-Schmidt laws relating $\Sigma_{\text {SFR }}$ and $\Sigma_{\text {dense }}$. However, LIRGs/ULIRGs and normal galaxies are not fully overlapping in this scatter plot (see Fig. 6 of GarcíaBurillo et al. 2012). In order to quantify if a dual fit applies to the power law, we split the sample into normal and IR luminous galaxies. A two-function power law fit with indices close to unity qualifies as a much better description of the star formation relation for the dense gas. This result is seen to be solid against the statistical biases inherent to this analysis (see discussion in Sect. 5 of García-Burillo et al. 2012).

The validity of a universal $M_{\text {dense }}-L_{\mathrm{HCN}(1-0)}^{\prime}$ conversion factor $\left(\alpha_{\mathrm{HCN}}\right)$ for normal galaxies and LIRGs/ULIRGs has been questioned by a number of works, which favor 

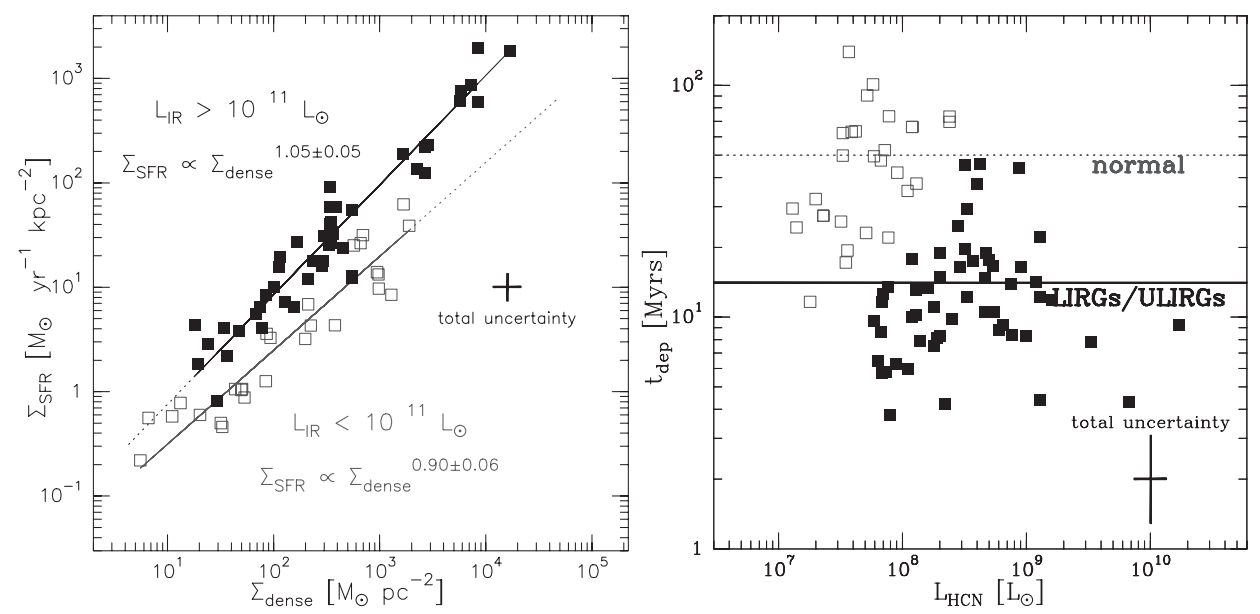

Figure 2. a) (left panel) Star-formation rate surface density, $\Sigma_{\mathrm{SFR}}$, as a function of dense molecular gas surface density, $\Sigma_{\text {dense }}$, obtained with the revised conversion factors discussed in Sect. 2. The power law fits to normal galaxies (gray line) and luminous infrared galaxies (black line) are shown. Symbols as in Fig. 1. b) (right panel) We show the revised depletion time scale as a function of $L_{\mathrm{HCN}}^{\prime}$ derived in normal and luminous infrared galaxies, as discussed in Sect. 2. The horizontal lines indicate the average value of the depletion time scale in normal galaxies $\left(\mathrm{t}_{\mathrm{dep}} \sim 50 \pm 5 \mathrm{Myr}\right)$ and LIRGs/ULIRGs $\left(\mathrm{t}_{\mathrm{dep}} \sim 14 \pm 1.4 \mathrm{Myr}\right)$.
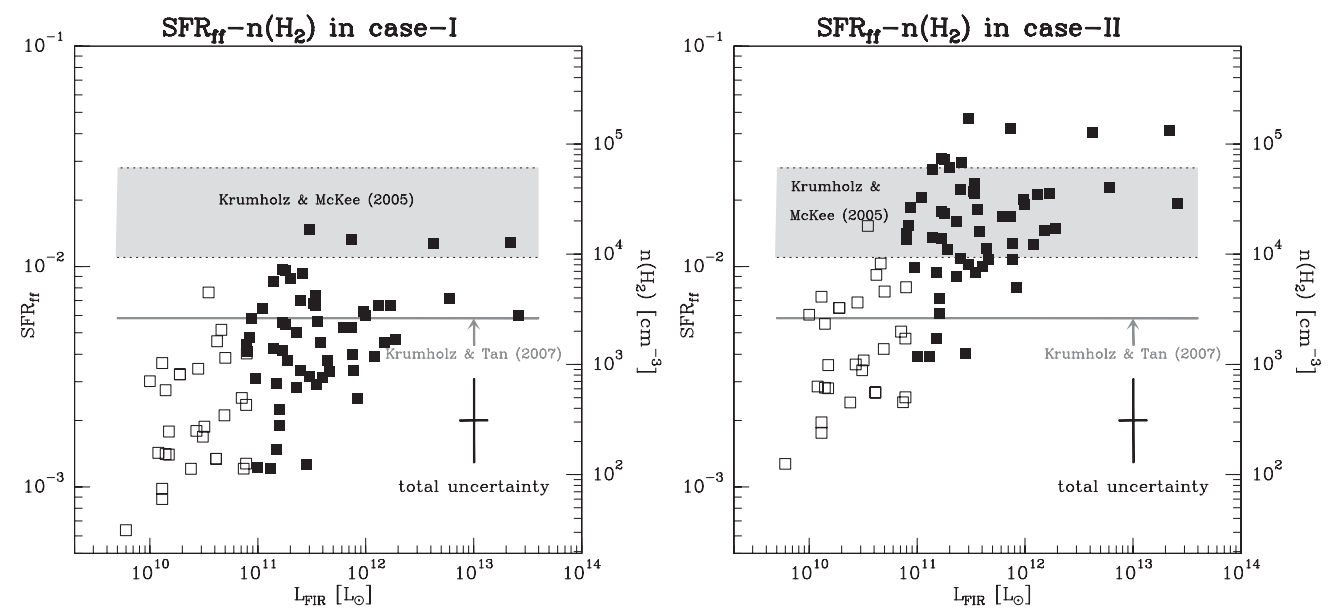

Figure 3. a) (left panel) The $\mathrm{SFR}_{\mathrm{ff}}$ (left $\mathrm{Y}$ axis) derived from the observed $\mathrm{SFE}_{\mathrm{dense}}$ in different populations of galaxies assuming a gas density for $\operatorname{HCN~} \mathrm{n}_{\mathrm{HCN}}\left(\mathrm{H}_{2}\right)=3 \times 10^{4} \mathrm{~cm}^{-3}$. We show on the right $\mathrm{Y}$ axis the value of $\mathrm{n}_{\mathrm{HCN}}\left(\mathrm{H}_{2}\right)$ derived from $\mathrm{SFE}_{\text {dense }}$ assuming a constant $\mathrm{SFR}_{\mathrm{ff}}=0.02$, a value within the range determined by the star formation model of Krumholz \& McKee (2005). We also highlight the average value of $\mathrm{SFR}_{\mathrm{ff}} \sim 0.0058$ determined by Krumholz \& Tan (2007). Symbols are as in Fig. 1. b) (right panel) Same as a) but obtained from the revised values of $\Sigma_{\text {dense }}$ and $\Sigma_{\mathrm{SFR}}$ discussed in Sect. 6 of García-Burillo et al. (2012).

lower $\alpha_{\mathrm{HCN}}$ in extreme starbursts compared to normal galaxies (e.g., Graciá-Carpio et al. 2008; see, however, Papadopoulos et al. 2012). If we account for the different conversion factors for $\mathrm{HCN}$ in extreme starbursts and for the unobscured star formation rate in normal galaxies the duality in star formation laws is enhanced, as illustrated in Fig. 2a. At the high end of $\Sigma_{\text {dense }}$ values ( a few $10^{4} \mathrm{M}_{\odot} \mathrm{pc}^{-2}$ ) the normal galaxy law underpredicts $\Sigma_{\mathrm{SFR}}$ in IR luminous galaxies by about an order of magnitude. Furthermore, 
within the range of gas surface densities shared by normal galaxies and LIRGs/ULIRGs, $\Sigma_{\text {dense }} \sim 210^{1}-210^{3} \mathrm{M}_{\odot} \mathrm{pc}^{-2}$, the factor 3 to 5 disagreement between the two laws is a factor $\sim 3-4$ times larger than the typical uncertainty on $\Sigma_{\mathrm{SFR}}$. This result extends the more extreme bimodal behavior of star formation laws, derived from CO molecular lines by two recent surveys (Daddi et al. 2010, Genzel et al. 2010), to the higher molecular densities probed by HCN lines. On average, the revised depletion time scales for the dense molecular gas show a significant difference between LIRGs/ULIRGs $\left(\mathrm{t}_{\mathrm{dep}} \sim 14 \pm 1.4 \mathrm{Myr}\right)$ and normal galaxies ( $\mathrm{t}_{\mathrm{dep}} \sim 50 \pm 5 \mathrm{Myr}$ ) (Fig. 2b).

\section{Star-formation laws: observations versus models}

García-Burillo et al. (2012) have used the new HCN observations presented above to test a key prediction of the star formation model of Krumholz \& McKee (2005): the constancy of $\mathrm{SFR}_{\mathrm{ff}}$, which is expected to be $\sim 0.02$ up to the density regime explored by $\mathrm{HCN}$ observations. Figure 3 a represents $\mathrm{SFR}_{\mathrm{ff}}$ as a function of $\mathrm{L}_{\mathrm{FIR}}$ obtained from the $\mathrm{SFE}_{\text {dense }}$ values derived with universal conversion factors for $\mathrm{L}_{\mathrm{FIR}}$ and $\mathrm{L}_{\mathrm{HCN}}$. To render a constant $\mathrm{SFR}_{\mathrm{ff}}$ compatible with the order of magnitude increase in $\mathrm{SFE}_{\mathrm{dense}}$, the density of HCN clouds has to change notably from normal galaxies to LIRGs/ULIRGs. Nevertheless, the values derived for $\mathrm{SFR}_{\mathrm{ff}}$ and/or $\mathrm{n}_{\mathrm{HCN}}\left(\mathrm{H}_{2}\right)$ are well below the expected range both for normal galaxies and LIRGs/ULIRGs. The use of revised conversion factors alleviates the problem only in LIRGs/ULIRGs (Fig. 3b). An overall satisfactory solution, which accounts for the observed trend in the $\mathrm{L}_{\mathrm{FIR}} / \mathrm{L}_{\mathrm{HCN}}$ luminosity ratios, is only found provided that: 1 ) $\mathrm{SFR}_{\mathrm{ff}} \sim 0.0035$ (i.e., a factor $\sim 6$ lower than the canonical value of $\sim 2 \%), 2) \mathrm{n}_{\mathrm{HCN}}\left(\mathrm{H}_{2}\right)$ increases with $\mathrm{L}_{\mathrm{FIR}}$ from $\sim 10^{4} \mathrm{~cm}^{-3}$ in normal galaxies to $\sim 10^{6} \mathrm{~cm}^{-3}$ in extreme starbursts, and 3) $\alpha_{\mathrm{HCN}}$ is $\sim 2-4$ times lower in LIRGs/ULIRGs. In summary, there is new observational evidence that the overall efficiency of star formation in the dense molecular gas is significantly lower than initially postulated by Krumholz \& McKee (2005). Furthermore, an agreement between observations and models requires a dramatic increase in the average gas densities from normal galaxies to extreme starbursts.

Multi-line observations are a key to constrain the conversion factors of the different molecular tracers that are commonly used as proxies for $\Sigma_{\text {gas }}$. These observations can also yield an estimate of volume gas densities. As recently argued by Krumholz et al. (2012) a simple, local, volumetric star formation law with $\mathrm{SFR}_{\mathrm{ff}} \sim 0.01$ can dissolve the apparent bimodality found by Genzel et al. (2010) if different scale-heights are assumed for the gas disks in normal galaxies and extreme starbursts. While this is a plausible explanation, the change in scale-heights required to make bimodality disappear in star formation laws remains to be observationally validated.

\section{References}

Alonso-Herrero, A., Rieke, G. H., Rieke, M. J., et al. 2006, ApJ, 650, 835

Daddi, E., Elbaz, D., Walter, F., et al. 2010, ApJ (Letters), 714, L118

Gao, Y. \& Solomon, P. M. 2004, ApJ, 606, 271

García-Burillo, S., Usero, A., Alonso-Herrero, A., et al. 2012, A\&A, 539, A8

Genzel, R., Tacconi, L. J., Graciá-Carpio, J., et al. 2010, MNRAS, 407, 2091

Graciá-Carpio, J., García-Burillo, S., Planesas, P., et al. 2008, A\&̛A, 479, 703

Krumholz, M. R. \& McKee, C. F. 2005, ApJ, 630, 250

Krumholz, M. R. \& Tan, J. C. 2007, ApJ, 654, 304

Krumholz, M. R., Dekel, A., \& McKee, C. F. 2012, ApJ, 745, 69

Papadopoulos, P. P., van der Werf, P., Xilouris, E., Isaak, K. G., \& Gao, Y. 2012, ApJ, 751, 10

Schmidt, M. 1959, ApJ, 129, 243 